\title{
Interaction between an Accelerated Mass in Straight Motion and a Hidden Energy Reservoir as a Strict Mathematical Consequence of Special Relativity
}

\author{
Andreas Trupp \\ Fachhochschule Muenster (University of Applied Science), Muenster, Germany \\ Email: andreas@andreastrupp.com
}

How to cite this paper: Trupp, A. (2022) Interaction between an Accelerated Mass in Straight Motion and a Hidden Energy Reservoir as a Strict Mathematical Consequence of Special Relativity. Journal of Modern Physics, 13, 16-33.

https://doi.org/10.4236/jmp.2022.131002

Received: November 23, 2021

Accepted: January 17, 2022

Published: January 20, 2022

Copyright $\odot 2022$ by author(s) and Scientific Research Publishing Inc. This work is licensed under the Creative Commons Attribution International License (CC BY 4.0).

http://creativecommons.org/licenses/by/4.0/

\section{(c) (i) Open Access}

\begin{abstract}
A. Einstein and H.A. Lorentz had found that the mass of an accelerated body traveling at relativistic velocity appears to depend on whether the acceleration is performed in the direction of motion or in a transverse direction. E.P. Epstein rejected this result in the "Annalen der Physik"; he rather postulated an additional force that turns up when the body is accelerated in the longitudinal direction. It can be shown that the concept of an increased longitudinal mass is based on a simple mathematical error. When correcting this error, it turns out that Epstein's additional, hidden force is indispensable in order to avoid an inner inconsistency of Special Relativity. It does most of the total work absorbed by the moving object, and is thus responsible for most of the increase in its energy (=mass), given the speed attained is relativistic. In other words: While the total force on the body needed to maintain a constant acceleration $a_{0}$ is " $\left(1-v^{2} / c^{2}\right)^{-1} m a_{0}=m_{0}\left(1-v^{2} / c^{2}\right)^{-3 / 2} a_{0}$ ", the technical force needed to maintain that acceleration amounts only to

" $m a_{0}=m_{0}\left(1-v^{2} / c^{2}\right)^{-1 / 2} a_{0}$ ". The total energy of two objects that undergo a symmetrical, elastic head-on collision is therefore not conserved during the collision, thus requiring the involvement of a hidden reservoir of energy. This result is confirmed by calculations that use the concept of momenergy. The phenomenon of an apparent disappearance of energy has been noticed in particle physics already (target-experiment), but its consequences have been ignored. Instead, an explanation has been given (reduced "energy of the center of mass") which is inconsistent and violates the relativity principle.
\end{abstract}

\section{Keywords}

Special Relativity, Longitudinal Mass, Transverse Mass, Momenergy, 
Target Experiment, Energy of the Center of Mass, Dark Energy

\section{Introduction}

1) In the past, Special Relativity has been subject to various attempts to modify it. Most attempts of a modification have also been an attempt of a refutation. This is because in those attempts the basic assumptions of Special Relativity, i.e., the relativity principle and the invariance of the speed of light, have been cast into doubt as regards their postulated reach.

For instance, J. Wang [1] (pp. 1615-1644) recently revived the more-than-acentury-old idea according to which an ether could exist which "sticks" to heavy celestial bodies like a viscous and sticky fluid does to the surface of a spoon, so that no "ether wind" can be felt on their surfaces. As a consequence, the speed of light would not always be the same. At a location far away from earth where the "ether wind" could not be neglected, it would be different from the speed measured in experiments on earth. But since Special Relativity is based on the rigorous assumption of the speed of light being invariant for any observer without any exceptions, Special Relativity would not be a valid description of nature if the "viscous liquid" theory regarding an ether were true. However, experiments have been performed the outcome of which was unfavorable for the theory of a "viscous and sticky ether". The first of these experiments was the Michelson-Gale experiment in 1925.

Another recent attempt of modifying Special Relativity was undertaken by N.H. Abramson [2] (pp. 471-478). He modified the relativistic factor $k$ which appears in the Lorentz-transformation by inserting the term $M /(M+m)$ in front of $v^{2} / c^{2}$ as a factor, and by defining $v$ as the speed of the center of gravity of the system of the two observers involved (who are thought to have mass). But when working backward from the so modified equations of the Lorentz transformation, at least one of the two basic (empirical) equations from which the Lorentz transformation is derived is altered. However, no empirical evidence is presented which would compel us to prefer the new basic equation(s) over the old one(s).

My article which I am presenting here is of a different kind. It is by no means an attempt to modify and refute the basic assumptions of Special Relativity. What it does is the following: it reveals some strict, physically surprising mathematical consequences of these basic assumptions, which have so far been overlooked. The reason for this failure lies in the fact that a parameter which turns up in one of the equations of Special Relativity has commonly been interpreted in a way which contradicts a presupposition. This inconsistency leads to mathematically wrong consequences. They can be found in all textbooks which mention the subject.

2) According to the Theory of Special Relativity, the mass of a body has increased when it has gathered speed. Mass is resistance offered against acceleration. One should expect that the resistance a moving body offers against accele- 
ration does not depend on the direction in which the acceleration is performed. But Einstein had to realize that the equations of Special Relativity DO make a difference regarding the direction of acceleration: the resistance is stronger when the acceleration is performed in the direction of motion, and is weaker in a transverse direction. Einstein therefore distinguished between a transverse and a longitudinal mass of a body in motion. He thus accepted (as did most physicists after him) the strange consequence that the directions of an applied force and that of the resulting acceleration deviate from each other. Whether or not this is justified shall be scrutinized in this article.

3) The nomenclature used is the following:

$a=$ acceleration of the object in the lab's unprimed frame of reference

$c=$ speed of light

$E=$ total energy $\mathrm{mc}^{2}$ of the moving object, which includes the energetic equivalent of its rest mass

$E_{\text {conv }}=$ energy thought to be available for conversion when two particles collide with each other

$$
\begin{aligned}
& F=\text { force } \\
& h=\text { parameter used to replace a complicated term } \\
& k=\text { relativistic factor, equal to }\left(1-v^{2} / c^{2}\right)^{-1 / 2} \\
& m_{0}=\text { rest mass of an object } \\
& m=\text { total mass of an object (rest mass plus relativistic mass) } \\
& p=\text { linear momentum of an object in the unprimed frame of reference } \\
& s=\text { distance (in the unprimed frame of reference) covered by a moving object } \\
& t=\text { time in the unprimed reference frame in which an object is moving } \\
& t a u=\text { proper time of a moving object } \\
& u^{\prime}=\text { velocity of an object measured in the primed reference frame (rest frame) }
\end{aligned}
$$
of an observer; the primed frame is moving in the unprimed reference frame at a velocity $v$

$u=$ velocity of an object in the unprimed reference frame; the object is not necessarily at rest in the primed frame, but can be in motion in the primed frame, its velocity there being $u^{\prime}$

$v=$ velocity at which the primed frame itself or an object at rest in the primed frame is moving in the unprimed reference frame

$W=$ work done (in the unprimed frame of reference) on an object when accelerating it

$x=$ direction in which an object is moving in the unprimed frame of reference

$y=$ direction perpendicular to the $x$ - and the $z$-direction

$z=$ direction perpendicular to the $x$ - and the $y$-direction

\section{The Distinction between Transverse and Longitudinal Mass}

In case a body-moving in straight and steady motion with a velocity $V_{x}$ along the positive $x$-axis of an unprimed coordinate system-is becoming subject to an 
accelerating force $F_{x}$ in the positive $x$-direction, that force is (according to Special Relativity):

$$
\begin{aligned}
& F_{x}=\frac{\mathrm{d} p_{x}}{\mathrm{~d} t}=\frac{\mathrm{d}\left[m(t) v_{x}(t)\right]}{\mathrm{d} t}=\frac{\mathrm{d}\left[k(t) m_{0} v_{x}(t)\right]}{\mathrm{d} t}=\frac{\mathrm{d}\left[\frac{m_{0} v_{x}(t)}{\sqrt{1-v_{x}^{2}(t) / c^{2}}}\right]}{\mathrm{d} t} \\
& =\left[\frac{m_{0}}{\left(1-v_{x}^{2}(t) / c^{2}\right)^{1 / 2}}+\frac{m_{0} v_{x}^{2}}{c^{2}\left(1-v_{x}^{2}(t) / c^{2}\right)^{3 / 2}}\right] a_{x}=\frac{m_{0}}{\left(1-v_{x}^{2}(t) / c^{2}\right)^{3 / 2}} a_{x}
\end{aligned}
$$

The term $p$ denotes the momentum of the body. The term $m$ stands for the time-dependent relativistic (total) mass of that body, which is equal to the product of its rest mass $m_{0}$ and the relativistic factor $k=\left(1-v^{2} / c^{2}\right)^{-1 / 2}$. In purely mathematical terms, a presupposition made in Equation (1) is:

$$
m=\frac{m_{0}}{\sqrt{1-v_{x}^{2} / c^{2}}}
$$

(In order to avoid confusion in connection with historical references, I will use the somewhat old-fashioned terms "rest mass" $m_{0}$ for objects at $v=0$, and "mass" $m$ for the total mass of an object, which, because of $m=E / c^{2}$, depends on rest mass and its kinetic energy.) The term $a=\mathrm{d} v / \mathrm{d} t$ denotes the acceleration of the body in the unprimed frame of reference. The sum that turns up in the second line is the result of an application of the quotient rule in differential calculus.

One realizes: when looking at the right-hand side of the second line of Equation (1), the total mass $m$ of the body appears to be equal to $m_{0}\left(1-v^{2} / c^{2}\right)^{-3 / 2}$, and not $m_{0}\left(1-v^{2} / c^{2}\right)^{-1 / 2}$ as was presupposed. This appears to be an inner inconsistency, which, fortunately, will dissolve further below.

In case an acceleration is taking place only in a direction transverse to the original $x$-direction of motion, namely in the $y$-direction, we find for the force:

$$
F_{y}=\frac{\mathrm{d} p_{y}}{\mathrm{~d} t}=\frac{\mathrm{d}\left[m v_{y}(t)\right]}{\mathrm{d} t}=\frac{\mathrm{d}\left[k m_{0} v_{y}(t)\right]}{\mathrm{d} t}=\frac{\mathrm{d}\left[\frac{m_{0} v_{y}(t)}{\sqrt{1-v_{\text {total }}^{2} / c^{2}}}\right]}{\mathrm{d} t}=\frac{m_{0} a_{y}}{\sqrt{1-v_{\text {total }}^{2} / c^{2}}}
$$

The result falls short of what is gained for the $x$-component. The relativistic total mass $m$ of the body and hence also the relativistic factor $k$ do not depend on time here. This is because none of the terms that show up on the right-hand side of the equation depend on time. Although the total velocity $v_{\text {total }}$ does increase a bit if the body is undergoing an accelation in the $y$-direction, this increase in total velocity vanishes because motion and acceleration are at right angle with respect to each other.

The same is true when it comes to an acceleration in the $z$-direction.

\section{The Mass-Energy-Equivalence Principle}

As regards the work $W$ done when accelerating the body at a constant rate $a_{x}$ 
(assuming the validity of $v=a t$ and of $s=1 / 2 a t^{2}$ ), we get because of Equation (1):

$$
\begin{aligned}
\mathrm{d} W & =F \mathrm{~d} s=\frac{m_{0}}{\left(1-v^{2} / c^{2}\right)^{3 / 2}} a_{x} \mathrm{~d}\left(\frac{1}{2} a_{x} t^{2}\right)=\frac{m_{0}}{\left(1-v^{2} / c^{2}\right)^{3 / 2}} a_{x} \mathrm{~d}\left(\frac{1}{2} a_{x} \frac{v^{2}}{a_{x}^{2}}\right) \\
& =\frac{m_{0}}{\left(1-v^{2} / c^{2}\right)^{3 / 2}} \frac{1}{2}(v \mathrm{~d} v+v \mathrm{~d} v)=\frac{m_{0}}{\left(1-v^{2} / c^{2}\right)^{3 / 2}} v \mathrm{~d} v
\end{aligned}
$$

and therefore:

$$
W=\int_{0}^{W(v)} \mathrm{d} W=\int_{0}^{s(v)} F \mathrm{~d} s=\int_{0}^{v} \frac{m_{0}}{\left(1-v^{2} / c^{2}\right)^{3 / 2}} v \mathrm{~d} v=\frac{m_{0}}{\left(1-v^{2} / c^{2}\right)^{1 / 2}} c^{2}-m_{0} c^{2}
$$

This equation - which constitutes the mass-energy-equivalence theorem-was set up by A. Einstein [3] (section 10) in 1905.

For a non-relativistic velocity of $v=0.01$ (expressed in dimensionless units of a fraction of c), Equation (4) yields a work of 0.00005000375 per unit of rest mass, whereas Newton's law yields a work of exactly 0.00005 .

It should be noted that the mass-energy-equivalence principle holds true on the basis of $\left(1-v^{2} / c^{2}\right)^{-3 / 2} m_{0} a$ being the total force (exerted on the body in order to accelerate it), and would not hold true if the total force were just $\left(1-v^{2} / c^{2}\right)^{-1 / 2} m_{0} a$.

The total energy of a moving body is obtained by adding the energy $m_{0} c^{2}$ of the rest mass to $W$. We then get:

$$
E=W+m_{0} c^{2}=\frac{m_{0}}{\left(1-v^{2} / c^{2}\right)^{1 / 2}} c^{2}
$$

\section{R.C. Tolman's Contribution to the Discussion}

1) The discrepancy between an acceleration in a longitudinal and in a transverse direction was recognized by A. Einstein [and, prior to that, by H.A. Lorentz [4] (Section 9)]. Einstein (see Ref. 3, Section 10) therefore distinguished between a "transverse" and a "longitudinal mass" (so did H.A. Lorentz). P.G. Bergmann [5] (Chapter VI, Eq. 6.70 and 6.71, p. 103/104) expressed this finding as follows:

"The coefficients of the acceleration on the right-hand sides of eqs. (6.70) and (6.71) are occasionally referred to as 'longitudinal mass' and 'transversal mass', respectively."

N. Dragon [6] (Chapter 3.4, p. 53) recently wrote:

"Inertia of fast particles is direction-dependent. In case the force acts at right angle with respect to $\mathrm{v}$, the acceleration is $\mathrm{d} v / \mathrm{d} t=\left(1-v^{2}\right)^{1 / 2} \mathrm{~F} / \mathrm{m}$; in the direction of the velocity the inertia of the particle is increased by the factor $1 /\left(1-v^{2}\right)$."

The conflict between $m=m_{0}\left(1-v^{2} / c^{2}\right)^{-1 / 2}$ and $m=m_{0}\left(1-v^{2} / c^{2}\right)^{-3 / 2}$ was highligted by R.C. Tolman [7] (pp. 375-380) in 1912: 
"On the basis of these accelerations, it has been usual to place the 'transverse mass' of a body moving with the velocity $u$ as equal to $m_{0}\left(1-u^{2} / c^{2}\right)^{-1 / 2}$ and its 'longitudinal' mass as equal to $m_{0}\left(1-u^{2} / c^{2}\right)^{-3 / 2}$, where $m_{0}$ is the mass of the body at rest and $c$ is the velocity of light. If, however, mass is a quantity to which a conservation law applies, the mass of a body cannot well be different in different directions; and it has been believed by Professor Lewis and the writer, that in general, without respect to direction, the expression $m_{0}\left(1-u^{2} / c^{2}\right)^{-1 / 2}$ is best suited for the mass of a moving body."

Tolman set out from the following equation:

$$
-\left(m_{1}+m_{2}\right) v=m_{1} u_{1}+m_{2} u_{2}=m_{1} \frac{u^{\prime}-v}{1-u^{\prime} v / c^{2}}+m_{2} \frac{-u^{\prime}-v}{1+u^{\prime} v / c^{2}}
$$

The terms $m_{1}$ and $m_{2}$ are the relativistic total masses of two bodies-that have equal mass when at rest-at a picked moment in time. At that very moment of interest, we imagine the two perfectly elastic masses to be in the process of head-on collision, and, since they are, at that moment, in contact with each other, they are decelerating. In the primed frame of reference, one of the two bodies has the momentary velocity $+u$, and the other one has the momentary velocity $-u$ '. In the unprimed frame of reference, the primed frame of reference moves to the left at constant speed $v$. If assuming that the sum of the two masses (=energies), viewed in the unprimed frame of reference, stays the same over time (kinetic energy is converted into what Tolman calls "elastic energy", and vice versa), the total momentum of the two bodies in the unprimed frame of reference must be $-\left(m_{1}+m_{2}\right) v$ at the (somewhat later) moment when the two colliding objects will have come to rest in the primed frame of reference. Because of the assumed invariance of the sum of the two masses, this statement is valid despite the fact that $m_{1}$ and $m_{2}$ refer to the masses as they present themselves at a different moment in time (namely a moment prior to coming to relative rest). Because of an assumed conservation of momentum, $-\left(m_{1}+m_{2}\right) v$ must equal $m_{1} u_{1}+m_{2} u_{2}$, that is, the total momentum (sum of momenta) at the moment of deceleration. Finally, $u_{1}$ and $u_{2}$ are replaced in accordance with the relativistic theorem of addition of velocities.

By mere algebra, Tolman transforms his equation into:

$$
\frac{m_{1}}{m_{2}}=\frac{\left(1-u_{2}^{2} / c^{2}\right)^{1 / 2}}{\left(1-u_{1}^{2} / c^{2}\right)^{1 / 2}}
$$

When imagining that $u_{1}$ is zero (so that $m_{1}=m_{0}$, with $m_{0}$ being the mass of one of the two objects when it is at rest in the unprimed frame, but is being equipped with some elastic energy), this equation turns into:

$$
m_{2}=\frac{m_{0}}{\left(1-u_{2}^{2} / c^{2}\right)^{1 / 2}}
$$

Tolman thus obtained the result that the mass of a body in motion is, at any 
moment, equal to $m_{0}\left(1-v^{2} / c^{2}\right)^{-1 / 2}$, regardless of whether or not it is, in the moment considered, being accelerated or in steady motion.

But this does not bring us any further (even if Tolman's assumptions of the conservation of total mass and of total momentum were correct). Instead, it brings us to where we started, that is, to the presupposition regarding the mass $m$ that we made in the first line of Equation (1).

2) Some years later, Tolman [8] (Chapter 6, Section 69, Eq. 68, pp. 76/77) seems to have abandoned his opinion. He now states that $F$ is equal to $m_{0}\left(1-u^{2} / c^{2}\right)^{-3 / 2} \mathrm{~d} u / \mathrm{d} t$, and tells his readers that $m_{0}\left(1-u^{2} / c^{2}\right)^{-3 / 2}$ "is sometimes spoken of as the expression for the longitudinal mass of a particle."

\section{The Need for an Additional, Hidden Force (First Postulated by P.S. Epstein)}

1) But given that the relationship $m=m_{0}\left(1-v^{2} / c^{2}\right)^{-1 / 2}$ is a presupposition made in Equation (1) and hence in the mass-energy-equivalence principle (Equation 4), the only way to avoid the mentioned inner inconsistency in Equation (1) and Equation (4) is the following: when a body is accelerated by means of an external, technical force, an additional, hidden force turns up. Then Equation (1) can be re-formulated as follows:

$$
F_{x}=\frac{m_{0}}{\left(1-v_{x}^{2} / c^{2}\right)^{3 / 2}} a_{x}=\frac{m_{0}}{\left(1-v_{x}^{2} / c^{2}\right)\left(1-v_{x}^{2} / c^{2}\right)^{1 / 2}} a_{x}=\frac{1}{1-v_{x}^{2} / c^{2}} m a_{x}
$$

Now $m$ is equal to $m_{0}\left(1-v^{2} / c^{2}\right)^{-1 / 2}$ even in the longitudinal direction, exactly as was presupposed in (1). The factor $\left(1-v^{2} / c^{2}\right)^{-1}$ which turns up on the right-hand side of (7) is an expression of the additional force, as it tells by which factor the net force is enlarged (in comparison with $F=m a$ ). One should therefore distinguish between a total force

$$
F_{\text {total }}=\frac{m_{0}}{\left(1-v_{x}^{2} / c^{2}\right)^{3 / 2}} a_{x}=\frac{1}{1-v_{x}^{2} / c^{2}} m a_{x}
$$

and a technical force $F_{\text {tech }}$ which we exert on the object by technical means:

$$
F_{\text {tech }}=\frac{m_{0}}{\left(1-v_{x}^{2} / c^{2}\right)^{1 / 2}} a_{x}=m a_{x}
$$

The difference between these two forces gives the additional, hidden force

$$
\begin{aligned}
F_{\text {hidden }} & =F_{\text {total }}-F_{\text {tech }}=\frac{m_{0}}{\left(1-v_{x}^{2} / c^{2}\right)^{3 / 2}} a_{x}-\frac{m_{0}}{\left(1-v_{x}^{2} / c^{2}\right)^{1 / 2}} a_{x} \\
& =\frac{m_{0}}{\left(1-v_{x}^{2} / c^{2}\right)^{1 / 2}} a_{x}\left(\frac{1}{1-v_{x}^{2} / c^{2}}-1\right)
\end{aligned}
$$

2) It was P.S. Epstein [9] (pp. 779, 783/784) who postulated such a force explicitly in the "Annalen der Physik" (1911):

"It is therefore of great advantage to introduce an additional force, ... This, 
is, for instance, accomplished by a compensation force $f_{x}^{k}=-\beta^{2} f_{x}$ acting in the $x$-direction, by virtue of which the $x$-component is weakened by a factor of $1-\beta^{2}, \ldots$ Moreover, one is thereby getting rid of the tensor mass; given that force exists, ... the mass is now $m_{0}\left(1-v^{2} / c^{2}\right)^{-1 / 2}$ in all directions."

Though Epstein conceived of a force whose direction was opposed to that of the technical force applied, he would surely not have raised concerns against a force that would act in the same direction as the technical force, since the "compensation force" he mentioned was only meant as an example. One has to confess that his example was a bad one. The force he took as an example would neutralize the accelerating force to some extent. As a net result, $F / a$ would not equal $m_{0}\left(1-v^{2} / c^{2}\right)^{-3 / 2}$ though this is what Equation (1) is saying. This would bring Relativity into trouble. Therefore the additional force must be of a different kind, with its direction not being opposed to the direction of the technical force applied, but sharing it. This is to be expanded in greater detail below.

\section{Determination of the Nature of Epstein's Additional, Hidden Force, and of the Source of the Energy It Conveys}

1) But if the mass of a moving body is $\left(1-v^{2} / c^{2}\right)^{-1 / 2}$ in all directions (regardless of whether or not it is subject to acceleration), then the additional, work-performing force (whose existence must then be acknowledged) cannot feed energetically from the same source as the technical force does: given the technical force $F_{\text {tech }}$ needed to maintain a fixed acceleration $a_{0}$ is $m a_{0}=m_{0}\left(1-v^{2} / c^{2}\right)^{-1 / 2} a_{0}$-and is not larger-then it falls short of $\left(1-v^{2} / c^{2}\right)^{-1} m a_{0}=m_{0}\left(1-v^{2} / c^{2}\right)^{-3 / 2} a_{0}$, which is the total force needed to maintain a fixed acceleration $a_{0}$. A work-performing hidden force-energetically fed from a hidden reservoir-is hence coming to the aid of the technical force.

As a consequence, the technical work needed to bring the object to a velocity $v$ only amounts to:

$$
\begin{aligned}
W_{\text {tech }} & =\int_{0}^{s(v)} F_{\text {tech }} \mathrm{d} s=\int_{0}^{s(v)} \frac{m_{0}}{\left(1-v^{2} / c^{2}\right)^{1 / 2}} a_{0} \mathrm{~d} s=\int_{0}^{v} \frac{m_{0}}{\left(1-v^{2} / c^{2}\right)^{1 / 2}} v \mathrm{~d} v \\
& =m_{0} c^{2}-m_{0} c^{2}\left(1-v^{2} / c^{2}\right)^{1 / 2}
\end{aligned}
$$

For a non-relativistic velocity of $v=0.01$ (expressed in dimensionless units of a fraction of c), Equation (11) yields an amount of work of 0.00005000126 per unit of rest mass. This falls short of what (4) is yielding, but exceeds the amount of work given by Newton's law by a small amount, quite as expected. The difference between the results of Equation (4) and Equation (11) (that is $0.00005000375-0.00005000126=0.00000000249$, or 224.1 Newtonmeter per $\mathrm{kg}$ ) is due to the work done by Epstein's hidden, additional force.

For a relativistic velocity that approaches $c$, Equation (4) yields an infinite 
amount of total work invested, whereas Equation (11) yields only $m_{0} c^{2}$ as the technical work spent on accelerating the object to nearly the speed of light. In that case, the hidden force has done much more work on the object than the technical force has, and is responsible for most of the increase in mass. Conversely, whenever a fast moving objects hits planet Earth, its kinetic energy cannot be larger than $m_{0} c^{2}$, and cannot possibly be above that (see below for further consequences in the field of Particle Physics).

From this follows: when a particle that has a rest mass $m_{0}$ and is traveling nearly at the speed of light is absorbed by a large, stationary system, the mass of that system thus increases by $2 m_{0}$. When a photon (rather than an object or a particle that has a rest mass) is absorbed by that large, stationary system, the mass of that system increases by $m$. The difference between the two cases can be attributed to the fact that the photon's rest mass is missing and exists only virtually.

2) To summarize: we must recognize that relativity postulates the emergence of an additional, hidden force which comes to the aid of the technical force applied to a body in order to accelerate it. The energy of that hidden, work-performing force is fed from a hidden reservoir in space. The flow of that hidden energy has its sink in the positively accelerated body, as has the ordinary energy that is conveyed by the technical force (but whose source is in a battery or a similar device). The increase in strength of the gravitational field generated by the fast moving body is thus somewhat larger than it should be on the basis of the absorbed technical work alone. When the fast moving body is decelerated, the energetic process is reversed, and the relativistic mass of the object transforms into a flow of energy whose sink is partly in the hidden reservoir.

Epstein's hidden force thus doesn't do any technical work on material objects, but is, as has just been said, capable of strengthening the gravitational field generated by the moving object. It should also lead to an increase in the centrifugal force the moving mass is subject to if, after a linear acceleration, the electrically charged object is exposed to a Lorentz force which bends its trajectory. This is no indirect departure from the rule according to which the hidden force cannot do any technical work, since the centrifugal force is incapable of doing work (due to its direction being at right angle to the motion of the object).

Thereby both $F_{\text {total }} / a=m_{0}\left(1-v^{2} / c^{2}\right)^{-3 / 2}$ and $F_{\text {tech }} / a=m_{0}\left(1-v^{2} / c^{2}\right)^{-1 / 2}$ present themselves as valid descriptions of nature.

\section{Neither Momenergy nor Energy Is Conserved during a Head-on Collision of Two Fast Moving Objects}

One should note that the total relativistic mass (generated by the combined work-performing actions of the technical and the hidden force) is subject to change during an elastic head-on collision of two alike bodies: the relativistic mass or energy that was generated by Epstein's hidden force prior to the head-on collision is not converted into elastic energy (when the two colliding objects come 
to relative rest), but flows off into the unknown where it had come from. Otherwise $F_{\text {tech }}$ would be identical with $F_{\text {total }}$.

In order to confirm this recognition, we turn our attention to momenergy (forgetting about collisions for a moment). Starting with Minkowski's line element of spacetime, we multiply both sides of Minkowski's equation by $m_{0}^{2} / \mathrm{d} \tau^{2}$ (with tau being proper time of the moving object). We then get:

$$
m_{0}^{2} \frac{\mathrm{d} \tau^{2}}{\mathrm{~d} \tau^{2}}=m_{0}^{2} \frac{\mathrm{d} t^{2}}{\mathrm{~d} \tau^{2}}-m_{0}^{2} \frac{\mathrm{d} x^{2}}{c^{2} \mathrm{~d} \tau^{2}}-m_{0}^{2} \frac{\mathrm{d} y^{2}}{c^{2} \mathrm{~d} \tau^{2}}-m_{0}^{2} \frac{\mathrm{d} z^{2}}{c^{2} \mathrm{~d} \tau^{2}}
$$

As a next step, we replace $\mathrm{d} \tau^{2}$ by $\mathrm{d} t^{2}\left(1-v^{2} / c^{2}\right)$. This is permissible because the two point events at the ends of a temporal interval we are considering occur at the same spatial location in the reference frame of the moving object. We then get:

$$
m_{0}^{2}=\frac{m_{0}^{2}}{1-v^{2} / c^{2}}-\frac{m_{0}^{2} v_{x}^{2}}{c^{2}\left(1-v^{2} / c^{2}\right)}-\frac{m_{0}^{2} v_{y}^{2}}{c^{2}\left(1-v^{2} / c^{2}\right)}-\frac{m_{0}^{2} v_{z}^{2}}{c^{2}\left(1-v^{2} / c^{2}\right)}
$$

or (see Equation (4a)):

$$
m_{0}^{2}=\frac{E^{2}}{c^{4}}-\left(\frac{p_{x}^{2}}{c^{2}}+\frac{p_{y}^{2}}{c^{2}}+\frac{p_{z}^{2}}{c^{2}}\right)
$$

The term $p$ denotes a momentum-component in the reference frame of the laboratory, $E$ denotes the total energy of the moving body. The right-hand side of Equation (13) or Equation (14) is known as the "momenergy" of the moving body [see for instance: E.F. Taylor, J.A. Wheeler [10] (Chapter 7.7, Eq. 7.3, p. 211)].

Similar to Tolman's reflections, we now imagine two alike bodies of the same rest mass which travel on straight paths, strictly in the $x$-direction of a Cartesian system of coordinates, the first one in the positive $x$-direction at speed $+V_{x}$, and the other one in the negative $x$-direction at speed $-V_{x}$ (equal and opposite velocities). Near the origin of coordinates, the two objects undergo an elastic head-on collision with each other. As long as the two objects are in contact with each other, they first decelerate, come to a full stop $\left(v_{x}=0\right)$, and pick up speed again (strictly in the positive and negative $x$-directions). As Tolman remarked, the rest mass $\mathrm{m}_{0}$ of each of the two bodies cannot be constant during the deceleration and acceleration, since both bodies are having what Tolman called "elastic energy" (see Reference 7, p. 377):

"Since at this time [of rest with respect to each other] both bodies are moving with the velocity $-\mathrm{v}$ we might suppose that $\mathrm{m}_{1}+\mathrm{m}_{2}$ equals $2 m_{0}\left(1-v^{2} / c^{2}\right)^{-1 / 2}$. This is not the case, however, since the bodies now possess additional elastic energy beyond that which they possess when at rest and not in contact."

The amount of that "elastic energy" is a function of $v$ in our case (with $0 \leq v<c$ ). This form of energy leads to an increase in the rest mass $m_{0}$ (of each of the two bodies), which, too, is therefore a function of $v$. Equation (13) thus 
converts into:

$$
m_{0}^{2}(v)=\frac{m_{0}^{2}(v)}{1-v^{2} / c^{2}}-\frac{m_{0}^{2}(v) v_{x}^{2}}{c^{2}\left(1-v^{2} / c^{2}\right)}-\frac{m_{0}^{2}(v) v_{y}^{2}}{c^{2}\left(1-v^{2} / c^{2}\right)}-\frac{m_{0}^{2}(v) v_{z}^{2}}{c^{2}\left(1-v^{2} / c^{2}\right)}
$$

The equation applies to each of the two bodies separately, whose behaviour is symmetrical.

When assuming-for a short moment-that the energy of each of the two bodies (total mass $m$ times $c^{2}$ ) stays constant during the collision even though its rest mass $m_{0}$ does not, we are setting up the following side-conditions:

$$
\mathrm{d} v_{y}=0, \quad \mathrm{~d} v_{z}=0, \quad \mathrm{~d}\left[\frac{m_{0}^{2}\left(v_{x}\right)}{1-v_{x}^{2} / c^{2}}\right]=\mathrm{d} E^{2}=0
$$

When forming the differential of Equation (15), we therefore get:

$$
\mathrm{d} m_{0}^{2}=2 m_{0} \mathrm{~d} m_{0}=-\mathrm{d}\left[\frac{m_{0}^{2} v_{x}^{2}}{c^{2}\left(1-v_{x}^{2} / c^{2}\right)}\right]
$$

$V_{x}$ shall now be considered as being a function of $m_{0}$. Re-arranging the last equation gives:

$$
-2 m_{0}=\frac{\mathrm{d}\left[\frac{m_{0}^{2} v_{x}^{2}}{c^{2}\left(1-v_{x}^{2} / c^{2}\right)}\right]}{\mathrm{d} m_{0}} \Rightarrow \frac{v_{x}^{2} / c^{2}}{1-v_{x}^{2} / c^{2}}=-1 \Rightarrow \frac{v_{x}^{2}}{c^{2}} \rightarrow \infty
$$

To elucidate: Since $\mathrm{d} m_{0}^{2} / \mathrm{d} m_{0}=2 m_{0}$, we know that $v^{2} /\left[c^{2}\left(1-v^{2} / c^{2}\right)\right]$ must equal or approach -1. [When $v^{2} /\left(c^{2}\left(1-v^{2} / c^{2}\right)\right)$ is substituted by the parameter $h\left[v\left(m_{0}\right)\right]$, Equation (18) converts into (using the product rule of differentiation): $-2=2 h+h^{\prime} m_{0}$. The variable $m_{0}$ doesn't have a fixed value during the collision, but can have any value within an interval. Moreover, the beginning (or end) of this interval can be as large or as small a number as desired, depending on how large or small a rest mass one chooses to start with. This makes sure that $h$ can have no value other than -1.] But $h$ can equal or approach -1 only when $v$ is many, many times larger than $c$.

If we set up the additional side condition: $v<\mathcal{c}$, we arrive at the conclusion that Equations (15), (16) and $v<c$ are incompatible with each other, and not all three of them can be valid in Special Relativity. Since we have no doubts concerning the physical validity of Equation (15) and of $v<c$, it is our assumption of $\mathrm{d} E^{2}=0$ (set up in Equation (16)) which we have to throw out. Explicitly, we arrive at:

$$
\mathrm{d} E \neq 0
$$

We have thereby obtained a confirmation of our result according to which there is a flow of energy into the unknown when the two objects decelerate during their head-on collision, and a flow of energy from the unknown into the objects when they are gathering speed thereafter. 


\section{The Disappearance of Energy (Mass) during "Target Experiments" in Particle Physics}

Particle physics has come across this phenomenon, too, but has so far not been able to explain it.

1) Let us consider particle collisions. The energy available for conversion in any collision (which includes the rest mass of the particle involved) shall be called $E_{c o n r}$ It is believed to be ( $c$ is set to unity and does not appear in the equation):

$$
\begin{aligned}
E_{\text {conv }} & =\sqrt{\left(E_{1}+E_{2}\right)^{2}-\left(p_{1_{x}}+p_{2_{x}}\right)^{2}-\left(p_{1_{y}}+p_{2_{y}}\right)^{2}-\left(p_{1_{z}}+p_{2_{z}}\right)^{2}} \\
& =\text { const }
\end{aligned}
$$

Equation (20) can be found in standard textbooks on particle physics like that of B. Povh, K. Rith, Ch. Scholz, F. Zetsche, W. Rodejohann [11] (Chapter 9, p. $123,124)$ or that of Ch. Berger [12] (Equations 1.39 and 1.40, p. 15). The term $E_{c o n v}$ on the left-hand side is considered (by particle physicists) as being an invariant. The invariance (if it exists) cannot be rooted in Equation (14). It is not an expression of the sum of the two momenergies of the two particles. This is because $\left(E_{1}+E_{2}\right)^{2}$ which appears in the equation is not equal to $\left(E_{1}^{2}+E_{2}^{2}\right)$. Likewise, $\left(p_{1}+p_{2}\right)^{2}$ is not equal to $\left(p_{1}^{2}+p_{2}^{2}\right)$. The reason why $E_{\text {conv }}$ is nevertheless thought to be invariant is the following: Equation (20) can be considered as being an expression of the supposed conservation both of energy and of momentum in the unprimed reference frame of the lab.

So far, so good. However, there is no reason why the alleged invariant should be identical with the energy available for conversion. Only $\left(E_{1}+E_{2}\right)$ alone can be an expression of that energy. It contains both the relativistic mass and the rest mass of the two particles involved in a collision. The total momentum of the two particles neither increases nor decreases that amount of energy, since the energy which could correspond to a momentum is already included in $E_{1}$ and/or $E_{2}$. Hence there is no justification for adding $-\left(p_{1_{x}}+p_{2_{x}}\right)^{2}-\left(p_{1_{y}}+p_{2_{y}}\right)^{2}-\left(p_{1_{z}}+p_{2_{z}}\right)^{2}$ under the root sign. We will come back to this point soon.

2) In what is called a "colliding-beam experiment", two particles of the same rest mass are moving at velocities $+u$ and $-u$ (equal and opposite), and collide with each (just as in Tolman's thought experiment). For such a collision, Equation (20) gives:

$$
E_{\text {conv }}=\sqrt{\left(E_{1}+E_{2}\right)^{2}}=2 E
$$

The energy available in that collision is $2 E$. The error contained in Equation (20), i.e., the existence of the summands $-\left(p_{1_{x}}+p_{2_{x}}\right)^{2}-\left(p_{1_{y}}+p_{2_{y}}\right)^{2}-\left(p_{1_{z}}+p_{2_{z}}\right)^{2}$, is neutralized here, simply because these summands are all zero.

But when one of the two particles (of equal mass) is at rest (this is the case in a so called "target experiment"), the energy available in such a collision according to Equation (20) is not $1 E+1 m_{0}$, but is much lower in amount. For Equation (20) then gives: 


$$
\begin{aligned}
E_{\text {conv }} & =\sqrt{\left(E_{1}+m_{0_{2}}\right)^{2}-p_{1_{x}}^{2}-p_{1_{y}}^{2}-p_{1_{z}}^{2}} \\
& =\sqrt{E_{1}^{2}+2 E_{1} m_{0_{2}}+m_{0_{2}}^{2}-p_{1_{x}}^{2}-p_{1_{y}}^{2}-p_{1_{z}}^{2}} \\
& =\sqrt{E_{1}^{2}+2 E_{1} m_{0}+m_{0}^{2}-E_{1}^{2}+m_{0}^{2}} \\
& =\sqrt{2 E_{1} m_{0}+2 m_{0}^{2}}<1 E_{1}+1 m_{0} \quad\left(\text { for } E_{1}>m_{0}, \text { i.e., for } u_{1}^{2}>0\right)
\end{aligned}
$$

The replacement of $-p_{x}^{2}-p_{y}^{2}-p_{z}^{2}$ by $-E^{2}+m_{0}^{2}$ in Equation (22) is justified by Equation (14). That is to say: our relativistic Equation (14) (which describes momenergy) now comes into play. Hence, the energy thought to be available for conversion is only $\left(2 E m_{0}+2 m_{0}^{2}\right)^{1 / 2}$.

As an example, we set $m_{0}=1$ and $E=10,000$ for a single (moving) particle. The energy available in a "colliding-beam experiment" thus is 20,000. For comparison, the energy available in the "target experiment" according to Equation (22) is only 141, much less than the energy of the particle which had been in motion before collision-and had had an energy of 10,000 then.

We realize: Equation (20) — and hence also Equation (22) - is the result of a desperate search for a way to account for observations made when a fast moving particle was hitting a bulk of stationary matter, with the particles generated by the impact having altogether much less energy than the impacting particle had. The sole purpose of the insertion of the summands $-\left(p_{1_{x}}+p_{2_{x}}\right)^{2}-\left(p_{1_{y}}+p_{2_{y}}\right)^{2}-\left(p_{1_{z}}+p_{2_{z}}\right)^{2}$ into Equation (20) has been to model the equation after the observed facts, and to reduce the yielded sum in case of a "target experiment". But doing an adaption like this is pure nonsense, as the subtraction of $p^{2}$ from $E^{2}$ is no physically meaningful method of determining the maximum energy available for conversation.

3) A common illustration of what is thought to be the deeper reason for Equation (22) is the following: it is believed that only what is called the "energy of the center of mass" is available for a conversion when it comes to a collision. Different from the situation in a "colliding-beam experiment" in which almost all sub-particles can be affected by a collision, only some of the sub-particles a proton is made up are believed to collide with each other when the collision of protons is a "target-collision", i.e., a collision with a target which is stationary. The rest of the sub-particles is thought of as not being affected by the "target collision".

There is, however, an obvious objection to this explanation: the distinction between a "colliding-beam experiment" and a "target experiment" is relative. In the reference of one of the two collision partners (in which that partner is at rest), all experiments are "target experiments". In another frame of reference which has just the right velocity $v$, both the particle at rest with respect to the lab and the particle in motion with respect to the lab have equal and opposite velocities $+u^{\prime}$ and $-u^{\prime}$. That is to say: in that frame of reference, all experiments are "colliding-beam experiments". But whether or not a fraction-which can exceed 90 percent or more-of the sub-particles penetrate each other (just as if the protons were made of intangible matter) is an absolute phenomenon which cannot 
depend on the frame of reference chosen for a description. Insofar as the existence of an absolute phenomenon is said to stand and fall with a certain reference frame (that of the lab), the relativity principle is violated.

Moreover, one should note that both Equation (21) and Equation (22) should be valid even when it comes to a small velocity of the moving particle. On top of this, Equation (22) has no upper limit for the rest mass of the particles. They can be cannon balls or bowling balls. But when a cannon ball moving at a speed of 10 $\mathrm{m} / \mathrm{sec}$ hits another cannon ball which is at rest, all sub-particles of the moving bowling ball come to rest, and are therefore affected by the collision without any exceptions. Hence, the true amount of energy available for conversion is $1 E+$ $1 m_{0}$, and not less.

4) Qualitatively, there is indeed an energetic difference between a symmetric collision ("colliding-beam experiment") and a target experiment: in a "colliding-beam experiment", energy flows from the two particles into a hidden reservoir when both particles are decelerating. However, when the resulting particles re-accelerate thereafter, the flow of energy is reversed, and energy from the hidden reservoir returns to the particles. The energy available for conversion can thus be as high as $2 E$. In a "target experiment", energy leaves the decelerating particle for the hidden reservoir. If the "target experiment" leads to a full stop of the moving particle without accelerating any particle other than in the form of thermal motion, the total energy available for a conversion into heat cannot be larger than $2 m_{0}$ (see above).

\section{The Directions of Applied Force and Resulting Acceleration}

As a consequence of the inconsistent assumption according to which the inert mass of a fast moving body depends on direction, authors usually arrive at the (wrong) conclusion that an acceleration of the body does not point in the same direction as the (technical) force that caused that acceleration. If, for instance, the force vector forms an angle of 45 with the velocity vector, the acceleration vector forms an angle whose size is between 45 and 90 . See for instance: N. Dragon (Reference 6, p. 53):

"In general, acceleration does not point in the direction of the applied force, ...”

Since common opinion has so far refrained from the idea that a hidden energy can be in possession of an inert mass so big that it is capable of bringing about a correct counter-force, the assumed non-coincidence of the two vectors must present a serious challenge to the principle of force and counter-force (and also to momentum conservation).

This challenge has so far been ignored. It can be met in the following way: presupposing (for reason of simplicity) the longitudinal component $F_{\text {tech-x }}=m_{0}\left(1-v^{2} / c^{2}\right)^{-1 / 2} a_{x}$ of the applied technical force is equal in magnitude to its transverse component by arrangement, the longitudinal component of the 
acceleration $a_{x}$ is as large as the transverse component of $a$. This follows from Equation (2), according to which the transverse component of the force is $F_{\text {tech-y }}=m_{0}\left(1-v^{2} / c^{2}\right)^{-1 / 2} a_{y}$. Hence the directions of the two vectors $F_{\text {tech }}$ and $a$ are one and the same, quite as they should.

\section{Confirmation of Epstein's Hidden Force by Similar Cases in Electromagnetism, Revealed by the Recognition That the Principle of Force and Counter-Force Is a Local One}

Surprisingly, the existence of a hidden force of that kind is confirmed by very simple reflections on some everyday phenomena:

Let us conceive of two equal spheres charged with electricity of the same sign. The two stationary spheres sit at a distance of 1 meter apart from each other. The electric field $E$ of each of the two spheres then exerts a repulsive electrostatic force on the other. At first sight, this alone appears to meet the principle of force and counter-force: a force felt by the first sphere is accompanied by a force in the opposite direction felt by the second sphere.

But this is not enough for the principle of force and counter-force to be observed. This is because that principle (like the principle of energy conservation) is a local one: the counter-force must turn up at the same location at which the force itself is attacking. This requirement has become indispensable after Special Relativity showed that the simultaneity of two events which are spatially separated from each other is relative, that is, dependent on the frame of reference chosen. Given force and counter-force have to appear simultaneously, the principle of force and counter-force thus can be obeyed in all possible frames of reference only if the spatial distance between the two locations is zero.

In electromagnetism, the Poynting vector, that is, the cross product of the vectors $E$ and $B$, plays an important role in connection with the principle of local momentum conservation and hence with that of force and counter-force. The Poynting-vector field makes flows of electromagnetic energy in space "visible". Moreover, when the Poynting vector undergoes changes with time, the momentum of the energy flow that it reveals does so, too, and thus a force (that may act as a counter-force with respect to another force) is revealed.

So far, so good. However, changes in the components of the Poynting vector cannot reveal a possible counter-force (exerted on the inert mass of the electromagnetic energy in the immediate vicinity of the sphere) if both the Poynting vector and the change in its components over time are zero. As regards our two charged spheres at rest, we simply have to imagine that their inert mass is very large. Despite the fact that a momentum transfer onto each of the two spheres is taking place, those spheres do not start to move visibly. Even after a considerably long interval of time, practically no motion of the spheres has been produced, and hence also no magnetic field $B$ (which will turn up as soon as a sphere is moving). Consequently, the Poynting vector, that is the cross product of $E$ times $B$, stays zero over time, and no change in momentum of the energy (=mass) of 
an electromagnetic field is brought about. That is to say: the counter-force must act on an object in the immediate vicinity of each sphere, but this object cannot be the mass of the electromagnetic field. The sphere that is receiving a momentum transfer must "push away" a hidden object (that exists in its immediate vicinity), instead.

The same is true if we replace the two charged spheres by two permanent magnets that repel or attract each other.

\section{The Challenge of the Proximity Principle by Mach's Principle When It Comes to Rotations, and How This Challenge Can Now Be Met}

Some important experiments in physics do not need structures like the LHC or LIGO, but require a cup of coffee only. If the coffee is brought into relative rotation with respect to the material of the cup, it is still undecided as to whether or not the liquid will rise along the walls of the cup. It will only rise in case the rotation of the liquid occurs relative to the distant stars as well. In that case, it does not even matter if there is a relative rotation between the liquid and the material of the cup.

We are thus confronted with "Mach's Principle", which reads: the inertial force on particles and bodies on earth and in the solar system is due to their acceleration relative to all matter residing outside the solar system. As regards the application of that principle to rotations, Mach's principle appears to be undoubtedly correct (as was pointed out by N. Graneau/P. Graneau [13], Chapter 7, p. 144). In E. Mach's [14] own words (Chapter II, sub-chapter VI 5, page 232):

“Newton's experiment with the rotating vessel of water simply informs us, that the relative rotation of the water with respect to the sides of the vessel produces NO noticeable centrifugal forces, but that such forces ARE produced by its relative rotation with respect to the mass of the earth and the other celestial bodies.”

But this is at odds with the proximity principle valid in physics as well. $M$. Planck [15] (Section 1, p. 2) formulated that principle as follows:

"According to it, when predicting chains of events to happen at a given location one does not need to care about what is going on somewhere else at other, finitely distant places, but may restrict oneself to causes in the immediate neighborhood; whereas, in case of believing in actions at a distance, one is, strictly speaking, compelled to check the whole universe for causes that may have a noticeable effect on the events that are to be determined by calculations."

The conflict between these two principles has widely been ignored, obviously because there seems to be no way of saving the proximity principle. However, the proximity principle can be saved by the recognition that the coffee in the cup, when being stirred with a spoon, does not rotate with respect to distant 
stars and galaxies, but with respect to the local "hidden energy" described. Though this energy cannot be attributed a direction or a speed of translational motion, its state does provide a reference for rotation.

It should be mentioned that H. Reichenbach [16] (Chapter III, Section 38The problem of rotation according to Einstein-p. 240) had tried to save the proximity principle in a similar, though very abstract way, by speaking of an "inertial field" that pervades the entire univserse:

"In this conception the fixed stars are at rest and determine an inertial field which pervades the entire space and lends inertia to every moving mass point."

But he couldn't explain why this "inertial field" was more than just a name for what should hopefully be detected in the future.

\section{Conclusions}

The main results are the following: while the total force on a body needed to maintain a constant acceleration $a_{0}$ is

$$
F=\frac{m}{1-v^{2} / c^{2}} a_{0}=\frac{m_{0}}{\left(1-v^{2} / c^{2}\right)^{3 / 2}} a_{0},
$$

the technical force needed to maintain that acceleration amounts only to

$$
F=\frac{m_{0}}{\left(1-v^{2} / c^{2}\right)^{1 / 2}} a_{0}
$$

It is therefore no surprise to find that the total energy of two (alike) objects that undergo a head-on collision is not conserved during the collision, thus requiring the involvement of a hidden reservoir of energy. That is to say: when treating the summand $E^{2} / c^{4}$ which appears in the momenergy equation

$$
m_{0}^{2}=\frac{E^{2}}{c^{4}}-\left(\frac{p_{x}^{2}}{c^{2}}+\frac{p_{y}^{2}}{c^{2}}+\frac{p_{z}^{2}}{c^{2}}\right)
$$

as a constant during the collision, we run into contradictions. The use of the equation

$$
E_{\text {conv }}=\sqrt{\left(E_{1}+E_{2}\right)^{2}-\left(p_{1_{x}}+p_{2_{x}}\right)^{2}-\left(p_{1_{y}}+p_{2_{y}}\right)^{2}-\left(p_{1_{z}}+p_{2_{z}}\right)^{2}}=\text { const }
$$

in particle physics, too, results in an apparent disappearance of energy, but is no valid way of describing this phenomenon.

The hidden energy which is indispensable in Special Relativity can be identified with the "dark energy" which turns up in General Relativity. Different from what has been believed since the existence of "dark energy" has been acknowledged in cosmology, "dark energy" thus does interact with other forms of energy at least indirectly (as it increases the strength of the gravitational field of fast moving objects), even though Special Relativity does not reveal any means how it could be harnessed as a source of technical work in cyclical processes. A. 
Trupp [17] showed that “dark energy" even interacts with Newton’s apple.

\section{Conflicts of Interest}

The author declares no conflicts of interest regarding the publication of this paper.

\section{References}

[1] Wang, J. (2019) Journal of Modern Physics, 10, 1615-1644. https://doi.org/10.4236/jmp.2019.1014107

[2] Abramson, N.H. (2018) Journal of Modern Physics, 9, 471-478. https://doi.org/10.4236/jmp.2018.93033

[3] Einstein, A. (1905) Annalen der Physik, 322, 891-921. https://doi.org/10.1002/andp.19053221004

[4] Lorentz, H.A. (1904) Electromagnetic Phenomena in System Moving with Any Velocity Less than That of Light. Proceedings of the Academy of Sciences of Amsterdam, Vol. 6, 809.

[5] Bergmann, P. (1976) Introduction to the Theory of Relativity. Revised Edition, Dover Publ., Mineola.

[6] Dragon, N. (2014) Geometrie der Relativitätstheorie. University of Hannover, Hannover.

[7] Tolman, R.C. (1912) Philosophical Magazine, 23, 375-380. https://doi.org/10.1080/14786440308637231

[8] Tolman, R.C. (1918) The Theory of the Relativity of Motion. University of California Press, Berkeley.

[9] Epstein, P.S. (1911) Annalen der Physik, 341, 779-795. https://doi.org/10.1002/andp.19113411404

[10] Taylor, E.F. and Wheeler, J.A. (1992) Spacetime Physics: Introduction to Special Relativity. 2nd Edition, W. H. Freeman, New York. https://doi.org/10.1119/1.17254

[11] Povh, B., Rith, K., Scholz, Ch., Zetsche, F. and Rodejohann, W. (2014) Teilchen und Kerne. 9th Edition, Springer, Berlin. https://doi.org/10.1007/978-3-642-37822-5

[12] Berger, Ch. (2014) Elementarteilchenphysik. 3rd Edition, Springer, Berlin. https://doi.org/10.1007/978-3-642-41753-5

[13] Graneau, P. and Graneau, N. (2006) In the Grip of the Distant Universe-The Science of Inertia. https://doi.org/10.1142/6087

[14] Mach, E. (1919) The Science of Mechanics. 4th Edition, The Open Court Publishing Company, Chicago and London.

[15] Planck, M. (1922) Einführung in die Theorie der Elektrizität und des Magnetismus.

[16] Reichenbach, H. (1958) The Philosophy of Space and Time. Dover Publ., Mineola.

[17] Trupp, A. (2019) Physics Essays, 32, 484-496. https://doi.org/10.4006/0836-1398-32.4.484 